\title{
Geometric properties of the ridge function manifold
}

\author{
Vitaly Maiorov
}

Received: 7 October 2005 / Accepted: 19 May 2008 /

Published online: 21 November 2008

(C) The Author(s) 2008. This article is published with open access at Springerlink.com

\begin{abstract}
We study geometrical properties of the ridge function manifold $\mathcal{R}_{n}$ consisting of all possible linear combinations of $n$ functions of the form $g(a \cdot x)$, where $a \cdot x$ is the inner product in $\mathbb{R}^{d}$. We obtain an estimate for the $\varepsilon$-entropy numbers in terms of smaller $\varepsilon$-covering numbers of the compact class $G_{n, s}$ formed by the intersection of the class $\mathcal{R}_{n}$ with the unit ball $B \mathcal{P}_{s}^{d}$ in the space of polynomials on $\mathbb{R}^{d}$ of degree $s$. In particular we show that for $n \leq s^{d-1}$ the $\varepsilon$-entropy number $H_{\varepsilon}\left(G_{n, s}, L_{q}\right)$ of the class $G_{n, s}$ in the space $L_{q}$ is of order $n s \log 1 / \varepsilon$ (modulo a logarithmic factor). Note that the $\varepsilon$-entropy number $H_{\varepsilon}\left(B \mathcal{P}_{s}^{d}, L_{q}\right)$ of the unit ball is of order $s^{d} \log 1 / \varepsilon$. Moreover, we obtain an estimate for the pseudo-dimension of the ridge function class $G_{n, s}$.
\end{abstract}

Keywords $\varepsilon$-entropy $\cdot$ Pseudo-dimension • Growth number • Ridge function manifold

Mathematics Subject Classifications (2000) $41 \mathrm{~A} 46$ • 41A30 • 26-04 • 54C70

\section{Introduction and main results}

Let $C\left(\mathbb{R}^{d}\right)$ be the space of all continuous functions on the space $\mathbb{R}^{d}$. Consider in $C\left(\mathbb{R}^{d}\right)$ the class of functions

$$
\mathcal{R}=\operatorname{span}\left\{g_{i}\left(a_{i} \cdot x\right): g_{i} \in C(\mathbb{R}), \quad a_{i} \in \mathbb{R}^{d}\right\},
$$

Communicated by Juan Manuel Peña.

V. Maiorov $(\bowtie)$

Department of Mathematics, Technion, Haifa, Israel

e-mail: maiorov@tx.technion.ac.il 
consisting of all possible linear combinations of continuous ridge functions of the form $g(a \cdot x), a \in \mathbb{R}^{d}$, where $a \cdot x$ is the inner product of vectors $a$ and $x$. Let $n$ be any natural number. Denote by

$$
\mathcal{R}_{n}=\left\{\sum_{i=1}^{n} g_{i}\left(a_{i} \cdot x\right): \quad g_{i} \in C(\mathbb{R}), a_{i} \in \mathbb{R}^{d}\right\},
$$

the subclass in $\mathcal{R}$ formed by all possible linear combinations of $n$ ridge functions.

The study of such a manifold $\mathcal{R}$ of ridge functions plays a central role in both pure and applied mathematics as is manifested in the series of works [3, 6$8,10,12$ ] (Temlyakov, unpublished manuscript) that concern the density of $\mathcal{R}$ in the space of continuous functions and the approximation of function classes by $\mathcal{R}$ (see also the survey of [9]).

Let $L_{q}=L_{q}(\Omega), 1 \leq q<\infty$, be the space of q-integrable functions on the unit cube $\Omega=[-1,1]^{d}$ with the norm $\|f\|_{L_{q}}=\left(\int_{\Omega}|f(x)|^{q} d x\right)^{1 / q}$. In the case $q=\infty$ the space $L_{\infty}(\Omega)$ consists of all function with the bounded norm $\|f\|_{L_{\infty}}=\operatorname{ess} \sup \{|f(x)|: x \in \Omega\}$. Denote by $B L_{q}=\left\{f:\|f\|_{L_{q}} \leq 1\right\}$ the unit ball in the space $L_{q}$. Given a natural number $s$ we denote by $\mathcal{P}_{s}^{d}$ the space of all real polynomials of degree at most $s$ on $\mathbb{R}^{d}$. Let $B_{q} \mathcal{P}_{s}^{d}=B L_{q} \cap \mathcal{P}_{s}^{d}$ be the unit ball in the space $\mathcal{P}_{s}^{d}$.

In the current work we study certain geometrical properties of the manifold $\mathcal{R}_{n}$, namely, we estimate the $\varepsilon$-entropy numbers in terms of smaller $\varepsilon$-covering numbers of the compact class $G_{n, s}=\mathcal{R}_{n} \cap B_{q} \mathcal{P}_{s}^{d}$ formed by the intersection of the class $\mathcal{R}_{n}$ with the unit ball in the space of polynomials of degree $s$ on $\mathbb{R}^{d}$. In particular we show that for $n \leq s^{d-1}$, the $\varepsilon$-entropy number $H_{\varepsilon}\left(G_{n, s}, L_{q}\right)$ of the class $G_{n, s}$ in the space $L_{q}$ is of order $n s \log _{2} 1 / \varepsilon$ (modulo a logarithmic factor). Note that the $\varepsilon$-entropy number $H_{\varepsilon}\left(B_{q} \mathcal{P}_{s}^{d}, L_{q}\right)$ of the unit ball $B_{q} \mathcal{P}_{s}^{d}$ is of order $s^{d} \log _{2} 1 / \varepsilon$. This result answers the question posed by Allan Pinkus: what is cardinality of the intersection of the ridge function manifold with the unit ball in the space of polynomials of a given degree?

Let $X$ be a Banach space and let $B=\{x \in X:\|x\| \leq 1\}$ the unit ball in $X$. Denote by $B(x, r)=r B+x$ the ball in $X$ of radius $r$ centered at the point $x$. All logarithms henceforth are taken with respect to 2. Let $F$ be some compact set in the space $X$. For any positive number $\varepsilon$ the $\varepsilon$-entropy of a set $F$ in the space $X$ represents the quantity $H_{\varepsilon}(F, X)=\log N_{\varepsilon}(F, X)$, where $N_{\varepsilon}(F, X)$ is the minimal number of elements in $F$ those forms an $\varepsilon$-net, i.e.

$$
N_{\varepsilon}(F, X)=\min \left\{N: \exists x_{1}, \ldots, x_{N} \in F \text { such that } F \subset \bigcup_{k=1}^{N} B\left(x_{k}, \varepsilon\right)\right\} .
$$

$N_{\varepsilon}(F, X)$ is called the $\varepsilon$-covering number of the set $F$. In the class $\mathcal{R}_{n}$ consider the intersection

$$
\mathcal{R}_{n, s}=\mathcal{R}_{n} \cap \mathcal{P}_{s}^{d}
$$

of the manifold $\mathcal{R}_{n}$ with the polynomial space $\mathcal{P}_{s}^{d}$. 
Theorem 1.1 Let $n, s \in \mathbb{N}, 1 \leq q \leq \infty$ and $0<\varepsilon<1$. Then the $\varepsilon$-entropy number of the class $\mathcal{R}_{n, s} \cap B L_{q}$ in the space $L_{q}$ satisfies the inequalities

1. if $n \leq s^{d-1}$, then

$$
c_{1} n s \leq \frac{H_{\varepsilon}\left(\mathcal{R}_{n, s} \cap B L_{q}, L_{q}\right)}{\log \frac{1}{\varepsilon}} \leq c_{2} n s l_{n, s},
$$

where $l_{n, s}=\log \frac{2 e s^{d-1}}{n}$,

2. if $n>s^{d-1}$, then

$$
c_{1}^{\prime} s^{d} \leq \frac{H_{\varepsilon}\left(\mathcal{R}_{n, s} \cap B L_{q}, L_{q}\right)}{\log \frac{1}{\varepsilon}} \leq c_{2}^{\prime} s^{d},
$$

where $c_{1}, c_{2}, c_{2}, c_{2}^{\prime}$ are constants depending only on $d$.

Consider another property of the manifold $\mathcal{R}_{n, s}$. For a given vector $h=$ $\left(h_{1}, \ldots, h_{m}\right) \in \mathbb{R}^{m}$ we define the sgn-valued vector $\operatorname{sgn} h=\left(\operatorname{sgn} h_{1}, \ldots, \operatorname{sgn} h_{m}\right)$, where $\operatorname{sgn} a=1$ for $a \geq 0$, and $\operatorname{sgn} a=-1$ for $a<0$. For a set $H \subset \mathbb{R}^{m}$, denote by $\operatorname{sgn} H$ the set of vectors $\{\operatorname{sgn} h: h \in H\}$. We denote by $H+a$ the set $\{h+a: h \in H\}$. For a finite set $Q$ we denote by $|Q|$ the cardinality of the set $Q$.

Definition 1 Let $R=\{r\}$ be a set of functions defined on $\mathbb{R}^{d}$. The VapnikChervonenkis dimension $\operatorname{dim}_{V C} R$ of the set $R$ is defined as the maximal natural number $m$ such that there exists a collection $\left\{\xi_{1}, \ldots, \xi_{m}\right\}$ in $\mathbb{R}^{d}$ for which the cardinality of the vector set $S=\left\{\left(\operatorname{sgn} r\left(\xi_{1}\right), \ldots \operatorname{sgn} r\left(\xi_{m}\right)\right): r \in R\right\}$ equals $2^{m}$. The quantity

$$
\operatorname{dim}_{p} R=\max _{f} \operatorname{dim}_{V C}(R+f),
$$

where $f$ runs over all functions defined on $\mathbb{R}^{d}$, is called the pseudo-dimension of the set $R$.

Theorem 1.2 Let $n, s \in \mathbb{N}$. Then there are a constants $c_{1}, c_{2}, c_{1}^{\prime}, c_{2}^{\prime}$ depending only on $d$, such that the pseudo-dimension of the set $\mathcal{R}_{n, s}$ satisfies the inequalities

1. if $n \leq s^{d-1}$, then $c_{1} n s \leq \operatorname{dim}_{p} \mathcal{R}_{n, s} \leq c_{2} n s l_{n, s}$,

2. if $n>s^{d-1}$, then $c_{1}^{\prime} s^{d} \leq \operatorname{dim}_{p} \mathcal{R}_{n, s} \leq c_{2}^{\prime} s^{d}$.

Henceforth we denote by $c, c_{i}, c_{i}^{\prime}, i=0,1, \ldots$ positive constants depending only on the parameter $d$. For two positive sequences $a_{n}$ and $b_{n}, n=0,1, \ldots$ we write $a_{n} \asymp b_{n}$ if there exist positive constants $c_{1}$ and $c_{2}$ such that $c_{1} \leq a_{n} / b_{n} \leq$ $c_{2}$ for all $n=0,1, \ldots$. 


\section{Estimating entropy by the growth number}

Let $B$ be any convex body in the $m$-dimensional vector space $\mathbb{R}^{m}$ which satisfies the symmetrical condition: if $x=\left(x_{1}, \ldots, x_{m}\right)$ belongs to $B$, then every point of the form $\left( \pm x_{1}, \ldots, \pm x_{m}\right)$ also belongs to $B$. We consider the linear normed space $l_{B}^{m}$ consisting of vectors from $\mathbb{R}^{m}$ with the unit ball $B$ and estimate the $\varepsilon$-entropy of a compact set $D$ by the growth number of $D$ which is defined as follows:

Definition 2 Given a set $D \subset \mathbb{R}^{m}$ define the number

$$
\operatorname{Gr}(D)=\max \left\{|\operatorname{sgn}(D-a)|: a \in \mathbb{R}^{m}\right\},
$$

which is called the growth number of the set $D$.

Theorem 2.1 Let $M$ be any set in the space $\mathbb{R}^{m}$ and $n=\log \operatorname{Gr}(M)$. Then for any $0<\varepsilon<1 / 2$ the $\varepsilon$-covering number of the intersection of the set $M$ with the unit ball $B$ satisfies the following inequality:

$$
N_{\varepsilon}\left(M \cap B, l_{B}^{m}\right) \leq\left(\frac{1}{\varepsilon}\right)^{c_{0} n},
$$

where $c_{0}$ is some absolute constant.

We start with proving a few auxiliary statements. Let $F$ be a compact body in the space $l_{B}^{m}$. We define the $\varepsilon$-capacity of the set $F$ as the quantity $H_{\varepsilon}^{c}\left(F, l_{B}^{m}\right)=$ $\log N_{\varepsilon}^{c}\left(F, l_{B}^{m}\right)$, where $N_{\varepsilon}^{c}\left(F, l_{B}^{m}\right)$ is the maximal number of points such that their pairwise-distances are at least $\varepsilon$, that is,

$$
N_{\varepsilon}^{c}\left(F, l_{B}^{m}\right)=\max \left\{N: \exists x_{1}, \ldots, x_{N} \in F \text { such that }\left\|x_{i}-x_{j}\right\| \geq \varepsilon, \forall i \neq j\right\} .
$$

$N_{\varepsilon}^{c}(F, X)$ is called the $\varepsilon$-capacity number of the set $F$.

Proposition 2.2 (see [4]) The $\varepsilon$-covering number and $\varepsilon$-capacity number of $F$ satisfy the following relationship:

$$
N_{2 \varepsilon}^{c}\left(F, l_{B}^{m}\right) \leq N_{\varepsilon}\left(F, l_{B}^{m}\right) \leq N_{\varepsilon}^{c}\left(F, l_{B}^{m}\right) .
$$

We briefly denote $N_{\varepsilon}\left(D, l_{B}^{m}\right)$ by $N_{\varepsilon}(D)$.

Lemma 2.3 Let $M$ be any set in the space $\mathbb{R}^{m}, a \in \mathbb{R}^{m}$, and $\operatorname{Gr}(M)=2^{n}$, $r$ be $a$ positive number and $B(a, r)=r B+a$ be a ball of radius $r$ with center $a$. Then for any positive number $\varepsilon$ there exists a point $a^{*} \in B(a, r)$ such that

$$
N_{\varepsilon}(M \cap B(a, r)) \leq 2^{n} N_{\varepsilon}\left(M \cap B\left(a^{*}, \frac{2}{3} r\right)\right) .
$$

Proof Introduce the set of vertices of the unit cube

$$
\Delta^{m}=\left\{\delta=\left(\delta_{1}, \ldots, \delta_{m}\right): \delta_{i}= \pm 1, i=1, \ldots, m\right\}
$$


in the space $\mathbb{R}^{m}$. Consider in $\Delta^{m}$ the subset $Q_{a}=\operatorname{sgn}(M-a)$. Since $\operatorname{Gr}(M)=$ $2^{n}$, then for every $a$ we have

$$
\left|Q_{a}\right| \leq 2^{n} .
$$

For every point $\delta \in \Delta^{m}$ we introduce the subset in the ball $B(a, r)$

$$
B(a, r, \delta)=\{x \in B(a, r): \operatorname{sgn}(x-a)=\delta\} .
$$

We have $B(a, r)=\bigcup_{\delta \in \Delta^{m}} B(a, r, \delta)$, and $B(a, r, \delta) \cap B\left(a, r, \delta^{\prime}\right)=\emptyset$ for any $\delta \neq$ $\delta^{\prime} \in \Delta^{m}$. From the definition of the set $Q_{a}$ we obtain

$$
M \cap B(a, r)=\bigcup_{\delta \in \Delta^{m}}[M \cap B(a, r, \delta)]=\bigcup_{\delta \in Q_{a}}[M \cap B(a, r, \delta)] .
$$

Then

$$
\begin{aligned}
N_{\varepsilon}[M \cap B(a, r)] & \leq N_{\varepsilon}\left[\cup_{\delta \in Q_{a}}(M \cap B(a, r, \delta))\right] \\
& \leq \sum_{\delta \in Q_{a}} N_{\varepsilon}[M \cap B(a, r, \delta)] \leq\left|Q_{a}\right| N_{\varepsilon}\left[M \cap B\left(a, r, \delta^{*}\right)\right],
\end{aligned}
$$

where $\delta^{*}$ is some vector in $\Delta^{m}$. Let $0<\lambda<1$ be some number and $a_{1}, \ldots, a_{s}$ a minimal $\lambda r$-net of the set $B\left(a, r, \delta^{*}\right)$. Then $s=N_{\lambda r}\left[B\left(a, r, \delta^{*}\right)\right]$. Therefore, we have the inequality

$$
N_{\varepsilon}\left[M \cap B\left(a, r, \delta^{*}\right)\right] \leq \sum_{i=1}^{s} N_{\varepsilon}\left[M \cap B\left(a_{i}, \lambda r\right)\right] \leq s N_{\varepsilon}\left[M \cap B\left(a_{i^{*}}, \lambda r\right)\right]
$$

for some $1 \leq i^{*} \leq s$. Put $\lambda=2 / 3$. By Proposition 2.2 we have

$$
\begin{aligned}
s & =N_{\lambda r}\left[B\left(a, r, \delta^{*}\right)\right] \leq N_{\lambda r}^{c}\left[B\left(a, r, \delta^{*}\right)\right] \leq \frac{V\left(B\left(a, r+\lambda r / 2, \delta^{*}\right)\right)}{V(B(0, \lambda r))} \\
& =\frac{2^{-m} V(B(a, r+\lambda r / 2))}{V(B(0, \lambda r))}=2^{-m}\left(\frac{r+\lambda r / 2}{\lambda r}\right)^{m}=1 .
\end{aligned}
$$

Hence the inequality (6) implies

$$
N_{\varepsilon}\left[M \cap B\left(a, r, \delta^{*}\right)\right] \leq N_{\varepsilon}\left[M \cap B\left(a_{i^{*}}, 2 r / 3\right)\right] .
$$

By the union of the inequalities (5), (4), (6) and (7) we have

$$
N_{\varepsilon}[M \cap B(a, r)] \leq 2^{n} N_{\varepsilon}\left[M \cap B\left(a, r, \delta^{*}\right)\right] \leq 2^{n} N_{\varepsilon}\left[M \cap B\left(a_{i^{*}}, 2 r / 3\right)\right]
$$

which proves the lemma.

Proof of Theorem 2.1 According to Lemma 2.3 there exists a sequence of points $a_{1}, \ldots, a_{k}$ belonging to the ball $B$ which satisfies the inequalities

$$
\begin{aligned}
N_{\varepsilon}[M \cap B] & \leq 2^{n} N_{\varepsilon}\left[M \cap B\left(a_{1}, 2 / 3\right)\right] \\
& \leq 2^{2 n} N_{\varepsilon}\left[M \cap B\left(a_{2},(2 / 3)^{2}\right)\right] \leq \ldots \leq 2^{k n} N_{\varepsilon}\left[M \cap B\left(a_{k},(2 / 3)^{k}\right)\right] .
\end{aligned}
$$


We choose $k$ such that $(2 / 3)^{k} \leq \varepsilon \leq(2 / 3)^{k-1}$. Then

$$
N_{\varepsilon}[M \cap B] \leq 2^{k n} N_{\varepsilon}\left[M \cap B\left(a_{k}, \varepsilon\right)\right]=2^{k n} \leq(1 / \varepsilon)^{c_{0} n},
$$

where $c_{0}=1+1 / \log (3 / 2)$. The theorem is proved.

\section{The entropy of polynomial manifolds}

Let $\alpha, \beta$ and $v$ be non-negative integers satisfying $v=\alpha+\beta$. Let $z=$ $\left(z_{1}, \ldots, z_{v}\right)$ be any point in the space $\mathbb{R}^{v}$. We represent the point $z$ as $z=(u, v)$ where $u=\left(u_{1}, \ldots, u_{\alpha}\right)$ and $v=\left(v_{1}, \ldots, v_{\beta}\right)$ are the corresponding projections of $z$ on the subspaces $\mathbb{R}^{\alpha}$ and $\mathbb{R}^{\beta}$.

Let $s$ be any natural number. Consider the matrix $Q_{m, \alpha}=\left(q_{i, j}\right)_{i=1, j=1}^{m, \alpha}$ which consists of polynomials $q_{i, j}=q_{i, j}(v)$ from the space $\mathcal{P}_{s}^{\beta}$. Construct the polynomials of degree $s+1$ on the space $\mathbb{R}^{v}$ as follows:

$$
p_{i}(z):=p_{i}(u, v):=\sum_{j=1}^{\alpha} u_{j} q_{i, j}(v), \quad i=1, \ldots, m .
$$

Introduce in the space $\mathbb{R}^{m}$ the polynomial manifold

$$
M:=M_{m, s, v, \alpha}:=\left\{\left(p_{1}(z), \ldots, p_{m}(z)\right): z \in \mathbb{R}^{\nu}\right\} .
$$

Theorem 3.1 Let $B$ be any convex body in the vector space $\mathbb{R}^{m}$ such that if $z=\left(z_{1}, \ldots, z_{m}\right)$ belongs to $B$, then every point of the form $\left( \pm z_{1}, \ldots, \pm z_{m}\right)$ also belongs to $B$. Let $v \leq m$. Then for any positive $\varepsilon$ the $\varepsilon$-covering number of the intersection of the manifold $M_{m, s, v, \alpha}$ with the body $B$ satisfies the inequality

$$
N_{\varepsilon}\left(M_{m, s, \nu, \alpha} \cap B, l_{B}^{m}\right) \leq\left(\frac{1}{\varepsilon}\right)^{c_{0} n_{m, s, v, \alpha}},
$$

where $n_{m, s, v, \alpha}=\log \left[(4 s)^{\beta}(v+1)^{\beta+2}\left(\frac{2 e m}{v}\right)^{v}\right]$.

Using Theorem 2.1 we have

$$
N_{\varepsilon}\left(M \cap B, l_{B}^{m}\right) \leq\left(\frac{1}{\varepsilon}\right)^{c_{0} \log \operatorname{Gr}(M)},
$$

where $\operatorname{Gr}(M)$ is the growth number of the manifold $M$. Thus in order to prove Theorem 3.1 we need to estimate the growth number $\operatorname{Gr}(M)$. This is closely related to the estimation of the number of connected components of polynomial manifolds as is done for instance in $[2,7,13,14]$.

Let $p$ be a real polynomial from the space $\mathcal{P}_{s}^{v}$, i.e., a polynomial on $v$ variables of degree at most $s$. The set of points $x \in \mathbb{R}^{v}$ on which a polynomial $p$ vanishes will be denoted by $Z(p)$. Let $D$ be a set in the space $\mathbb{R}^{v}$. We denote by $\operatorname{NCC}(D)$ the number of connected components of the set $D$. Let $p_{1}, \ldots, p_{m}$ 
be a collection of $m$ real polynomials from $\mathcal{P}_{s}^{v}$ and consider the finite set of sgn-valued vectors

$$
E\left(p_{1}, \ldots, p_{m}\right)=\left\{\left(\operatorname{sgn} p_{1}(z), \ldots, \operatorname{sgn} p_{m}(z)\right): z \in \mathbb{R}^{v}\right\} .
$$

In order to estimate the growth number $\operatorname{Gr}(M)$ we need an estimate on the cardinality of $E\left(p_{1}, \ldots, p_{m}\right)$. Introduce in the space $\mathbb{R}^{v}$ the domains $D=\bigcup_{i=1}^{m} Z\left(p_{i}\right)$ and $D^{\prime}=\mathbb{R}^{v} \backslash D$. We denote by $\operatorname{sgn} p(z)=\left(\operatorname{sgn} p_{1}(z), \ldots\right.$, $\left.\operatorname{sgn} p_{m}(z)\right)$. The vector function $\operatorname{sgn} p(z)$ is constant on any connected component of the domain $D^{\prime}$. Therefore the cardinality of the set $E\left(p_{1}, \ldots, p_{m}\right)$ does not exceed the number $N C C\left(D^{\prime}\right)$ of connected components of the domain $D^{\prime}$. Hence we now estimate the number $\operatorname{NCC}\left(D^{\prime}\right)$.

Theorem 3.2 Let $v \leq m$ and let $p_{1}, \ldots, p_{m}$ be the polynomials defined in (8). Then the number of connected components of the domain $D^{\prime}=\mathbb{R}^{v} \backslash \bigcup_{i=1}^{m} Z\left(p_{i}\right)$ satisfies the inequality

$$
\operatorname{NCC}\left(D^{\prime}\right) \leq(4 s)^{\beta}(v+1)^{\beta+2}\left(\frac{2 e m}{v}\right)^{v} .
$$

Proof We use the following inequality of Warren (see [14], Th. 1,2): there exist positive numbers $\delta_{1}, \ldots, \delta_{m}$ such that the number of connected components of the set $D^{\prime}$ satisfies the inequality

$$
\operatorname{NCC}\left(D^{\prime}\right) \leq \sum_{J} \sum_{\varepsilon_{j}= \pm 1, j \in J} \operatorname{NCC}\left(\bigcap_{j \in J} Z\left(p_{j}+\varepsilon_{j} \delta_{j}\right)\right),
$$

where $J$ runs over all subsets of the set $\{1, \ldots, m\}$, and $\varepsilon_{j}$ with $j \in J$ taking all possible values \pm 1 .

Let $r_{1}(v), \ldots, r_{n}(v)$ be any $n$ polynomials in the variable $v \in \mathbb{R}^{\beta}$ and let $\operatorname{deg} r_{i}$ be the degree of the polynomial $r_{i}$. We set $g=\operatorname{deg} r_{1}+\ldots+\operatorname{deg} r_{n}$. Consider in the space $\mathbb{R}^{\beta}$ the manifold

$$
R=\left\{v: r_{1}(v) \geq 0, \ldots, r_{n}(v) \geq 0\right\}
$$

Lemma 3.3 (Milnor [5], Th.3) The number $\mathrm{NCC}(R)$ of connected components of the set $R$ satisfies

$$
\mathrm{NCC}(R) \leq \frac{1}{2}(2+g)(1+g)^{\beta-1}
$$

From Milnor [5] it directly follows that the number of connected components, $\mathrm{NCC}(R)$, is bounded from above by rank $H^{*}(R)$ which is the rank of full cohomology group $H^{*}(R)$. Milnor obtained the estimate: rank $H^{*}(R) \leq$ $\frac{1}{2}(2+g)(1+g)^{\beta-1}$. 
Fix a subset $J$ from the set $\{1, \ldots, v\}$. Using Lemma 3.3 we estimate the numbers

$$
\operatorname{NCC}\left(\bigcap_{j \in J} Z\left(p_{j}+\varepsilon_{j} \delta_{j}\right)\right)
$$

Without loss of generality we may take $J=\{1, \ldots, k\}$, and $\varepsilon_{1}=\cdots=\varepsilon_{k}=$ -1 , where $k$ is the cardinality of the subset $J$.

Lemma 3.4 For any $1 \leq k \leq v$ and positive numbers $\delta_{1}, \ldots, \delta_{k}$, the following inequality holds

$$
\operatorname{NCC}\left(\bigcap_{i=1}^{k} Z\left(p_{i}-\delta_{i}\right)\right) \leq(4 s)^{\beta}(k+1)^{\beta+1} .
$$

Proof Consider the system of $k$ linear equations

$$
\left\{\begin{array}{c}
q_{11}(v) u_{1}+\ldots+q_{1 \alpha}(v) u_{\alpha}=\delta_{1} \\
\vdots \\
q_{k 1}(v) u_{1}+\ldots+q_{k \alpha}(v) u_{\alpha}=\delta_{k}
\end{array}\right.
$$

in the variables $u_{1}, \ldots, u_{\alpha}$, with coefficients $q_{i j}(v)$ and constants $\delta_{i}$. For any fixed $v$, denote by $D_{l}(v)$ the sum of squares of all minors of order $l$ of the matrix $\left(q_{i j}(z)\right)_{i=1 j=1}^{k, \alpha}$, and by $\overline{D_{l+1}}(v)$ the sum of squares of all minors of order $l+1$ of the extended matrix

$$
\left(\begin{array}{cccc}
q_{11}(v) & \ldots & q_{1 \alpha}(v) & \delta_{1} \\
\vdots & \ddots & \vdots & \vdots \\
q_{k 1}(v) & \ldots & q_{k \alpha}(v) & \delta_{k}
\end{array}\right)
$$

It follows from a theorem of Kronecker-Capelli that the set $V$ of all vectors $v \in \mathbb{R}^{\beta}$ for which there exists a solution to the system (12) may be expressed as

$$
V=\bigcup_{l=1}^{k-1} V_{l}, \quad V_{l}=\left\{v \in \mathbb{R}^{\beta}: D_{l}(v)>0, \overline{D_{l+1}}(v)=0\right\} .
$$

Since the $q_{i j}(v)$ are polynomials of degree $s$ then $D_{l}(v)$ and $\overline{D_{l+1}}(v)$ are polynomials of degree $2 s l$ and $2 s(l+1)$, respectively. From the continuous dependence of the solutions of (12) on the coefficients $q_{i j}(v)$, when $v$ runs over some connected component of $V_{l}$, it follows that $\operatorname{NCC}\left(\bigcap_{l=1}^{k} Z\left(p_{l}-\delta_{l}\right)\right)=$ $\mathrm{NCC}(V)$. From (13) we have

$$
\operatorname{NCC}\left(\bigcap_{l=1}^{k} Z\left(p_{l}-\delta_{l}\right)\right)=\operatorname{NCC}(V) \leq \sum_{l=1}^{k-1} \operatorname{NCC}\left(V_{l}\right) .
$$


Note that for any $l$ a set $V_{l}$ may be represented as the set of solutions of the system of inequalities

$$
D_{l}(v)>0, \quad-\overline{D_{l+1}}(v) \geq 0 .
$$

We claim that $\mathrm{NCC}\left(V_{l}\right) \leq(4 s(k+1))^{\beta}$. Indeed, assume that $N C C\left(V_{l}\right) \geq$ $\mu+1$, where $\mu=(4 s(k+1))^{\beta}$. Then there exist $\mu+1$ disjoint components $Q_{1}, \ldots, Q_{\mu+1}$ of the set $V_{l}$. For each $1 \leq i \leq \mu+1$, choose a point $w_{i}^{*}$ in $Q_{i}$. Put $\gamma=\min _{i} D_{l}\left(w_{i}^{*}\right)$, and note that $\gamma>0$. Consider the set in $\mathbb{R}^{\beta}$

$$
V_{l}^{\prime}=\left\{v: D_{l}(v) \geq \gamma,-\overline{D_{l+1}}(v) \geq 0\right\} .
$$

From Lemma 3.3 it follows that the number of connected components of the set $V_{l}^{\prime}$ satisfies the inequality $\operatorname{NCC}\left(V_{l}^{\prime}\right) \leq \frac{1}{2}(2+g)(1+g)^{\beta-1}$, where $g=$ $\operatorname{deg} D_{l}+\operatorname{deg} \overline{D_{l+1}}$. Since $g \leq 2 s l+2 s(l+1) \leq 4 s(k+1)$ we have

$$
\operatorname{NCC}\left(V_{l}^{\prime}\right) \leq \frac{1}{2}(2+4 s(k+1))(1+4 s(k+1))^{\beta-1} \leq(4 s(k+1))^{\beta}=\mu .
$$

On the other hand, since $V_{l}^{\prime} \cap Q_{i} \neq \emptyset$ for all $i=1, \ldots, \mu+1$, and $Q_{1}, \ldots, Q_{\mu+1}$ do not intersect, then

$$
\operatorname{NCC}\left(V_{l}^{\prime}\right) \geq \operatorname{NCC}\left(V_{l}\right) \geq \mu+1
$$

yielding a contradiction. Hence $\mathrm{NCC}\left(V_{l}\right) \leq(4 s(k+1))^{\beta}$. From here and (14) we obtain

$$
\operatorname{NCC}\left(\bigcap_{l=1}^{k} Z\left(p_{l}-\delta_{l}\right)\right) \leq \sum_{l=1}^{k-1} \operatorname{NCC}\left(V_{l}\right) \leq \sum_{l=1}^{k-1}(4 s(k+1))^{\beta} \leq(4 s)^{\beta}(k+1)^{\beta+1} .
$$

Lemma 3.4 is proved.

We now continue the proof of Theorem 3.2. From the inequality of (11) and Lemma 3.4 one obtains the following estimate for the number of connected components of the set $D^{\prime}=\mathbb{R}^{v} \backslash \bigcup_{i=1}^{m} Z\left(p_{i}\right)$

$$
\begin{aligned}
\operatorname{NCC}\left(D^{\prime}\right) & \leq \sum_{J} \sum_{\left\{\varepsilon_{j}: j \in J\right\}}(4 s)^{\beta}(k+1)^{\beta+1} \\
& \leq(4 s)^{\beta}(\alpha+\beta+1)^{\beta+1} \sum_{k=1}^{v}\left(\begin{array}{c}
m \\
k
\end{array}\right) 2^{k} \\
& \leq 2^{v}(4 s)^{\beta}(v+1)^{\beta+1} \sum_{k=1}^{v} \frac{m^{k}}{k !} \leq(4 s)^{\beta}(v+1)^{\beta+2}\left(\frac{2 e m}{v}\right)^{v},
\end{aligned}
$$

where we make use of the condition $\alpha+\beta=v \leq m$. Theorem 3.2 is proved.

Proof of Theorem 3.1 We estimate the growth number

$$
\operatorname{Gr}(M)=\max \left\{|\operatorname{sgn}(M-a)|: a \in \mathbb{R}^{m}\right\}
$$


of the manifold $M$. Let $a=\left(a_{1}, \ldots, a_{m}\right)$ be a fixed point in $\mathbb{R}^{m}$. Consider in the space $\mathbb{R}^{v}$ the domains $D_{a}=\bigcup_{i=1}^{m} Z\left(p_{i}-a_{i}\right)$ and $D_{a}^{\prime}=\mathbb{R}^{v} \backslash D_{a}$. Since the cardinality of the set $\operatorname{sgn}(M-a)$ does not exceed the number of connected components of the set $D_{a}^{\prime}$ then

$$
\operatorname{Gr}(M) \leq \max _{a \in \mathbb{R}^{v}} \operatorname{NCC}\left(D_{a}^{\prime}\right) .
$$

We have $v \leq m$. Therefore, from Theorem 3.2 for any $a \in \mathbb{R}^{v}$ we obtain

$$
\operatorname{NCC}\left(D_{a}^{\prime}\right) \leq(4 s)^{\beta}(v+1)^{\beta+2}\left(\frac{2 e m}{v}\right)^{v} .
$$

Combining the inequalities (16), (17) and (10) we obtain the estimate for the $\varepsilon$-covering number

$$
N_{\varepsilon}\left(M \cap B, l_{B}^{m}\right) \leq\left(\frac{1}{\varepsilon}\right)^{c_{0} n},
$$

where $n=\log \left[(4 s)^{\beta}(v+1)^{\beta+2}\left(\frac{2 e m}{v}\right)^{\nu}\right]$. Theorem 3.1 is proved.

\section{Proof of the main theorems}

Let $s$ be any natural number. Introduce in the interval $[-1,1]$ the collection of $4 s$ points $\xi_{i}=\cos \frac{(2 i-1) \pi}{8 s}, i=1, \ldots, 4 s$. Set $m=(4 s)^{d}$. Consider in the cube $\Omega=[-1,1]^{d}$ the finite lattice of points

$$
\Xi_{m}=\left\{\xi=\left(\xi_{i_{1}}, \ldots, \xi_{i_{d}}\right): i_{1}, \ldots, i_{d}=1, \ldots, 4 s\right\} .
$$

Let $l_{q}^{m}$ be the normed space of functions consisting of polynomials $P$ of the space $\mathcal{P}_{s}^{d}$ with the norm

$$
\|P\|_{l_{q}^{m}}=\left(\frac{1}{m} \sum_{\xi \in \Xi_{m}}|P(\xi)|^{q}\right)^{1 / q} .
$$

Denote by $B_{q}^{m}$ the unit ball in the space $l_{q}^{m}$. Consider in $\mathbb{R}^{m}$ the subset

$$
\mathcal{R}_{n, s}^{m}=\left\{\{P(\xi)\}_{\xi \in \Xi_{m}}: P \in \mathcal{R}_{n, s}\right\}
$$

which is the restriction of functions of $\mathcal{R}_{n, s}$ to the lattice $\Xi_{m}$.

Proposition 4.1 Let $1 \leq q \leq \infty$. Then there is a constant $c$ depending only on $d$ such that for any positive number

$$
N_{\varepsilon}\left(\mathcal{R}_{n, s} \cap B L_{q}, L_{q}\right) \leq N_{c \varepsilon}\left(\mathcal{R}_{n, s}^{m} \cap B l_{q}^{m}, l_{q}^{m}\right) .
$$

Proof Let $P$ be any polynomial from the space $\mathcal{P}_{s}^{d}$. Then the following inequality is true

$$
\|P\|_{L_{q}} \leq c\|P\|_{l_{q}^{m}}
$$


Indeed, by a change of variables $x_{k}=\cos t_{k}, k=1, \ldots, d$, we have

$$
\|P\|_{L_{q}}^{q}=\int_{\Omega}|P(x)|^{q} d x=\int_{[0, \pi]^{d}}|P(\cos t)|^{q} w(t) d t \leq \int_{[0, \pi]^{d}}|P(\cos t)|^{q} d t,
$$

where $\cos t=\left(\cos t_{1}, \ldots, \cos t_{d}\right)$ and $w(t)=\prod_{k=1}^{d} \sin t_{k}$. From the known relation (see [15]) we have

$$
\int_{[0, \pi]^{d}}|P(\cos t)|^{q} d t \leq \frac{c}{m} \sum_{\xi \in \Xi_{m}}|P(\xi)|^{q} .
$$

Thus, the inequality (19) is proved. Note that (19) directly implies the inequality of (18).

Proposition 4.2 Consider in the set $\mathcal{R}_{n, s}$ the subset

$$
\mathcal{R}_{n, s}^{*}=\left\{\sum_{i=1}^{n} \pi_{i}\left(a_{i} \cdot x\right): \quad \pi_{i} \in \mathcal{P}_{s}^{1}, a_{i} \in \mathbb{R}^{d}\right\},
$$

where $\pi_{i}$ are any univariate polynomials of degree at most s. Then $\mathcal{R}_{n, s}=\mathcal{R}_{n, s^{*}}^{*}$

By the Weierstrass theorem we have $\mathcal{R}_{n}=\overline{\bigcup_{k \geq s} \mathcal{R}_{n, k}^{*}}$, where $\bar{A}$ is the closure of the function set $A$ in the space of continuous functions on the ball $B^{d}$. Therefore,

$$
\mathcal{R}_{n, s}=\overline{\bigcup_{k \geq s} \mathcal{R}_{n, k}^{*}} \cap \mathcal{P}_{s}^{d} .
$$

Since the set $\left(\bigcup_{k \geq s} \mathcal{R}_{n, k}^{*}\right) \bigcap \mathcal{P}_{s}^{d}$ is not empty then

$$
\overline{\bigcup_{k} \mathcal{R}_{n, k}^{*}} \bigcap \mathcal{P}_{s}^{d}=\overline{\left(\bigcup_{k} \mathcal{R}_{n, k}^{*}\right) \bigcap \mathcal{P}_{s}^{d}}
$$

Therefore,

$$
\mathcal{R}_{n, s}=\overline{\left(\bigcup_{k} \mathcal{R}_{n, k}^{*}\right) \bigcap \mathcal{P}_{s}^{d}}=\overline{\bigcup_{k}\left(\mathcal{R}_{n, k}^{*} \bigcap \mathcal{P}_{s}^{d}\right)}
$$

We show that for every $k \geq s$ the equality $\mathcal{R}_{n, k}^{*} \cap \mathcal{P}_{s}^{d}=\mathcal{R}_{n, s}^{*}$ holds. Indeed, if $k=s$ then that is obvious. Let $k>s$ and let $\pi(x)=\sum_{i=1}^{n} \pi_{i}\left(a_{i} \cdot x\right)$ be any polynomial from the set $\mathcal{R}_{n, k}^{*} \cap \mathcal{P}_{s}^{d}$ where $\pi_{i} \in \mathcal{P}_{k}^{1}$ and $a_{i} \in \mathbb{R}^{d}$. For every $i$ we can represent the polynomial by $\pi_{i}\left(a_{i} \cdot x\right)=\sum_{j=0}^{k} c_{i j}\left(a_{i} \cdot x\right)^{j}$. Since the degree of polynomial $\pi$ is equal to $s$, then $c_{i j}=0$ for any $i$ and $s+1 \leq j \leq k$. Therefore the polynomial $\pi$ belongs to $\mathcal{R}_{n, s}^{*}$. Hence, $\mathcal{R}_{n, k}^{*} \cap \mathcal{P}_{s}^{d} \subseteq \mathcal{R}_{n, s}^{*}$. The inclusion $\mathcal{R}_{n, k}^{*} \cap \mathcal{P}_{s}^{d} \supseteq \mathcal{R}_{n, s}^{*}$ is obvious. Thus, $\mathcal{R}_{n, s}=\mathcal{R}_{n, s}^{*}$. 
Proposition 4.3 Let $l_{B}^{m}$ be the $m$-dimensional normed space $\mathbb{R}^{m}$ with a unit ball $B$. Then the $\varepsilon$-covering number $N_{\varepsilon}\left(B, l_{B}^{m}\right)$ of the ball $B$ satisfies

$$
(4 \varepsilon)^{-m} \leq N_{\varepsilon}\left(B, l_{B}^{m}\right) \leq(\varepsilon / 3)^{-m} .
$$

It follows from Proposition 2.2 that

$$
N_{2 \varepsilon}^{c}\left(B, l_{B}^{m}\right) \leq N_{\varepsilon}\left(B, l_{B}^{m}\right) \leq N_{\varepsilon}^{c}\left(B, l_{B}^{m}\right),
$$

where $N_{\varepsilon}^{c}\left(B, l_{B}^{m}\right)$ is the $\varepsilon$-capacity number of the ball $B$. Denote by $V(D)$ the Lebesgue volume of a set $D$. Then we have the obvious inequalities:

$$
N_{\varepsilon}^{c}\left(B, l_{B}^{m}\right) \leq \max \left\{n: V((1+\varepsilon) B)>n V\left(\frac{\varepsilon}{2} B\right)\right\}
$$

and

$$
N_{\varepsilon}^{c}\left(B, l_{B}^{m}\right) \geq \max \{n: V((1-\varepsilon) B)>n V(\varepsilon B)\} .
$$

Therefore, since $V(\varepsilon B)=\varepsilon^{m} V(B)$ we obtain

$$
(2 \varepsilon)^{-m} \leq N_{\varepsilon}^{c}\left(B, l_{B}^{m}\right) \leq(\varepsilon / 3)^{-m} .
$$

From here and (21) the statement of Proposition 4.3 directly follows.

Proof of Theorem 1.1 First we prove the right-hand inequalities in (1) and (2). Assume that $n \leq s^{d-1}$. From Proposition 4.1 we have

$$
N_{\varepsilon}\left(\mathcal{R}_{n, s} \cap B L_{q}, L_{q}\right) \leq N_{c \varepsilon}\left(\mathcal{R}_{n, s}^{m} \cap B l_{q}^{m}, l_{q}^{m}\right) .
$$

Using Theorem 2.1 we obtain

$$
N_{c \varepsilon}\left(\mathcal{R}_{n, s}^{m} \cap B l_{q}^{m}, l_{q}^{m}\right) \leq\left(\frac{1}{\varepsilon}\right)^{c_{0} \log \operatorname{Gr}\left(\mathcal{R}_{n, s}^{m}\right)},
$$

where $\operatorname{Gr}\left(\mathcal{R}_{n, s}^{m}\right)$ is the growth number of the manifold $\mathcal{R}_{n, s}^{m}$ and $c_{0}$ is some absolute constant.

Given $s, n$ and $d$ we define $m=(4 s)^{d}, \alpha=(s+1) n, \beta=d n$ and $v=\alpha+\beta$. We show that the manifold $\mathcal{R}_{n, s}^{m}$ may be represent by the form (9), (8). By Proposition 4.2 we have

$$
\mathcal{R}_{n, s}^{m}=\left\{\left\{P(\xi)=\sum_{k=1}^{n} \pi_{k}\left(a_{k} \cdot \xi\right)\right\}_{\xi \in \Xi_{m}}: \pi_{k} \in \mathcal{P}_{s}^{1}, a_{k} \in \mathbb{R}^{d}\right\} .
$$

We enumerate all points $\xi$ from the lattice $\Xi_{m}$ by $\xi_{1}, \ldots, \xi_{m}$. For fixed $i$ we consider the value

$$
P\left(\xi_{i}\right)=\sum_{k=1}^{n} \pi_{k}\left(a_{k} \cdot \xi_{i}\right)=\sum_{k=1}^{n} \sum_{l=0}^{s} c_{k, l}\left(a_{k} \cdot \xi_{i}\right)^{l} .
$$

of the polynomial $P$ in the point $\xi_{i}$ and denote $P\left(\xi_{i}\right)$ by $Q_{i}(c, a)$, that is consider the polynomial $Q_{i}$ in variables $c$ and $a$.

Enumerate the variables $\left\{c_{k, l}\right\}$, where $k=1, \ldots, n, l=0, \ldots, s$ by $\left\{u_{j}\right\}, j=$ $1, \ldots, \alpha, \alpha=n(s+1)$. 
Let $a_{k, 1}, \ldots, a_{k, d}$ be the coordinates of vector $a_{k}$. We enumerate the variables $\left\{a_{k, r}\right\}$, where $k=1, \ldots, n, r=1, \ldots, d$ by $\left\{v_{j}\right\}, j=1, \ldots, \beta, \beta=d n$.

Let $i$ is a fixed index. If the index $j$ correspond to given indexes $k$ and $l$ then we denote the functions $\left(a_{k} \cdot \xi_{i}\right)^{l}$ by $q_{i, j}(v)$. Then we can rewrite the polynomial $Q_{i}(c, a)$ by the form

$$
Q_{i}(c, a):=p_{i}(u, v):=\sum_{j=1}^{\alpha} u_{j} q_{i, j}(v) .
$$

Thus, we represent (see (9), (8)) the manifold $\mathcal{R}_{n, s}^{m}$ by the form

$$
M:=M_{m, s, v, \alpha}:=\left\{\left(p_{1}(u, v), \ldots, p_{m}(u, v)\right):(u, v) \in \mathbb{R}^{v}\right\} .
$$

Since $n \leq s^{d-1}$ then it is easy to see that $v \leq m$. Then according to inequalities (16) and (17) we have the estimate for the growth number

$$
\operatorname{Gr}\left(\mathcal{R}_{n, s}^{m}\right)=\operatorname{Gr}(M) \leq(4 s)^{\beta}(v+1)^{\beta+2}\left(\frac{2 e m}{v}\right)^{v} .
$$

By a straightforward computation we obtain

$$
\operatorname{Gr}\left(\mathcal{R}_{n, s}^{m}\right) \leq\left(\frac{2 e s^{d-1}}{n}\right)^{c_{1} s n},
$$

where $c_{1}=4 d(2 d+1)$. Set $c=c_{0} c_{1}$. Using the inequalities (22), (23) and (24) we then obtain

$$
N_{\varepsilon}\left(\mathcal{R}_{n, s} \cap B L_{q}, L_{q}\right) \leq\left(\frac{1}{\varepsilon}\right)^{c_{0} \log \operatorname{Gr}\left(\mathcal{R}_{n, s}^{m}\right)} \leq\left(\frac{1}{\varepsilon}\right)^{\operatorname{cns} \log \left(\frac{2 e^{d}-1}{n}\right)} .
$$

Thus the right-hand inequality in (1) is proved.

We prove the right-hand inequality in (2). Let $n>s^{d-1}$. Denote by $\mathcal{Q}_{s}^{d}$ the subspace in $\mathcal{P}_{s}^{d}$ consisting of all homogeneous polynomials of degree $s$. We know (see [7]) that

$$
\mathcal{P}_{s}^{d}=\mathcal{R}_{n, s}
$$

for any $n \geq \operatorname{dim} \mathcal{Q}_{s}^{d}$. Since (see [11]) $\operatorname{dim} \mathcal{Q}_{s}^{d}=\left(\begin{array}{c}s+d-1 \\ s\end{array}\right) \asymp s^{d-1}$, then

$$
N_{\varepsilon}\left(\mathcal{R}_{n, s} \cap B L_{q}, L_{q}\right) \leq N_{\varepsilon}\left(\mathcal{P}_{s}^{d} \cap B L_{q}, L_{q}\right) .
$$

Let $\mathcal{P}_{s}^{d}\left(\Xi_{m}\right)$ be the restriction of functions of the space $\mathcal{P}_{s}^{d}$ to the lattice $\Xi_{m}$. We have $\operatorname{dim} \mathcal{P}_{s}^{d} \asymp s^{d}$. Applying the inequality (19) and Proposition 4.3 we have

$$
N_{\varepsilon}\left(\mathcal{P}_{s}^{d} \cap B L_{q}, L_{q}\right) \leq N_{c \varepsilon}\left(\mathcal{P}_{s}^{d}\left(\Xi_{m}\right) \cap B l_{q}^{m}, l_{q}^{m}\right) \leq\left(\frac{1}{\varepsilon}\right)^{c^{\prime} s^{d}} .
$$

Thus, from (25) and (27) the right-hand inequalities in (2) directly follow.

Now we prove the left-hand inequalities in (1) and (2). Let $A=\left\{a_{1}, \ldots, a_{n}\right\}$ be a fixed collection of points from $\mathbb{R}^{d}$. Consider the linear subset in the set $\mathcal{R}_{n}$,

$$
\mathcal{R}_{n}(A)=\left\{\sum_{i=1}^{n} g_{i}\left(a_{i} \cdot x\right): g_{i} \in C(\mathbb{R})\right\},
$$


which is a linear space. Let $n \leq s^{d-1}$. Then $\frac{1}{d !} n s \leq \frac{s^{d}}{d !} \leq \operatorname{dim} \mathcal{P}_{s}^{d}$. Construct the sets $\mathcal{R}_{n, s}(A)=\mathcal{R}_{n}(A) \cap \mathcal{P}_{s}^{d}$ and

$$
\mathcal{R}_{n, s}^{*}(A)=\left\{\sum_{i=1}^{n} \pi_{i}\left(a_{i} \cdot x\right): \quad \pi_{i} \in \mathcal{P}_{s}^{1}\right\} .
$$

We can assume that the collection of points $a_{1}, \ldots, a_{n}$ is chosen such that the dimension $\mu=\operatorname{dim} \mathcal{R}_{n, s}^{*}(A)$ of the linear space $\mathcal{R}_{n, s}^{*}(A)$ belongs to the interval $\left[\frac{1}{d !} n s, \operatorname{dim} \mathcal{P}_{s}^{d}\right]$.

By analogy with Proposition 4.2 we can show that $\mathcal{R}_{n, s}^{*}(A)=\mathcal{R}_{n, s}(A)$. Also we have $\mathcal{R}_{n, s}^{*}(A) \subset \mathcal{R}_{n, s}$. Hence

$$
N_{\varepsilon}\left(\mathcal{R}_{n, s} \cap B L_{q}, L_{q}\right) \geq N_{\varepsilon}\left(\mathcal{R}_{n, s}^{*}(A) \cap B L_{q}, L_{q}\right) .
$$

Using Propositions 4.2, 4.3 and 2.2 we obtain

$$
N_{\varepsilon}\left(\mathcal{R}_{n, s}^{*}(A) \cap B L_{q}, L_{q}\right) \geq\left(\frac{1}{\varepsilon}\right)^{c \mu} .
$$

Since $\mu \geq c_{1} n s$, where $c_{1}=\frac{1}{d !}$, then the left-hand inequality in (1) is proved.

If $n>s^{d-1}$ then using the embedding (26) we have $\mathcal{P}_{c s}^{d} \subset \mathcal{R}_{n, s}^{*}(A)$ with some absolute constant $c$. Thus, we obtain $\mu \geq \operatorname{dim} \mathcal{P}_{c s}^{d} \asymp s^{d}$. Therefore,

$$
N_{\varepsilon}\left(\mathcal{R}_{n, s}^{*}(A) \cap B L_{q}, L_{q}\right) \geq\left(\frac{1}{\varepsilon}\right)^{c_{1} s^{d}},
$$

that is the left-hand inequality in (2) is also proved. Theorem 1.1 has now been completely proved.

Proof of Theorem 1.2 Assume that $n \leq s^{d-1}$. Set $\mu=\operatorname{dim} \mathcal{P}_{s}^{d}$. Let $\Xi$ be any finite set of points in $\mathbb{R}^{d}$. Denote by $\mathcal{R}_{n, s}^{\Xi}$ the restriction of the set $\mathcal{R}_{n, s}$ on $\Xi$. Then the following inequality holds:

$$
\begin{aligned}
\operatorname{dim}_{p} \mathcal{R}_{n, s} & =\max \left\{k: \exists \Xi, a \text { with }|\Xi|=k \text { and } a \in \mathbb{R}^{k} \text { s.t. }\left|\operatorname{sgn}\left(\mathcal{R}_{n, s}^{\Xi}-a\right)\right|=2^{k}\right\} \\
& \leq \log \max _{\Xi:|\Xi| \leq \mu} \operatorname{Gr}\left(\mathcal{R}_{n, s}^{\Xi}\right) .
\end{aligned}
$$

In analogy to the inequality of (24) we can show that

$$
\operatorname{Gr}\left(\mathcal{R}_{n, s}^{\Xi}\right) \leq\left(\frac{2 e s^{d-1}}{n}\right)^{c_{1} s n}
$$

for any $\Xi$ with $|\Xi| \leq \mu$. Thus,

$$
\operatorname{dim}_{p} \mathcal{R}_{n, s} \leq c_{1} n s \log _{2} \frac{2 e s^{d-1}}{n} .
$$


Let $n>s^{d-1}$. We know (see [1]) that the pseudo-dimension $\operatorname{dim}_{p} L$ of a linear finite-dimensional subspace $L$ coincides with the dimension $\operatorname{dim} L$ of $L$. Therefore,

$$
\operatorname{dim}_{p} \mathcal{R}_{n, s} \leq \operatorname{dim}_{p} \mathcal{P}_{s}^{d}=\operatorname{dim} \mathcal{P}_{s}^{d} \asymp s^{d} .
$$

From (29) and (30). the right-hand inequalities of Theorem 1.2 are proved.

Prove the left-hand inequalities. Let $n \leq s^{d-1}$. Let $A=\left\{a_{1}, \ldots, a_{n}\right\}$ be a collection of points from $\mathbb{R}^{d}$ such that $\operatorname{dim} \mathcal{R}_{n, s}(A) \geq$ cns with some constant $c$. Since $\mathcal{R}_{n, s}(A)$ is a linear subspace, then

$$
\operatorname{dim}_{p} \mathcal{R}_{n, s} \geq \operatorname{dim}_{p} \mathcal{R}_{n, s}(A)=\operatorname{dim} \mathcal{R}_{n, s}(A) \geq \text { cns. }
$$

If $n>s^{d-1}$ then for some set $A=\left\{a_{1}, \ldots, a_{n}\right\}$ the polynomial space $\mathcal{P}_{s}^{d}$ belongs to $\mathcal{R}_{n, s}$. Thus,

$$
\operatorname{dim}_{p} \mathcal{R}_{n, s} \geq \operatorname{dim}_{p} \mathcal{R}_{n, s}(A)=\operatorname{dim} \mathcal{P}_{s}^{d} \asymp s^{d} .
$$

Theorem 1.2 has been completely proved.

Open Access This article is distributed under the terms of the Creative Commons Attribution Noncommercial License which permits any noncommercial use, distribution, and reproduction in any medium, provided the original author(s) and source are credited.

\section{References}

1. Anthony, M., Bartlett, P.L.: Neural Network Learning: Theoretical Foundations. Cambridge University Press, Cambridge (1999)

2. Karpinski, M., Macintyre, A.J.: Polynomial bounds for VC dimension of sigmoidal neural networks. In: Proc. 27th ACM Synp. Thy. of Computing, pp. 200-208, June 1995

3. Lin, V., Ya., Pinkus, A.: Fundamentality of ridge functions. J. Approx. Theory 75, 295-311 (1993)

4. Kolmogorov, A.N., Tikhomirov, V.M.: $\varepsilon$-entropy and $\varepsilon$-capacity of sets in functional spaces. In: Shirayev, A.N. (ed.) Selected Works of A.N. Kolmogorov, vol. 3. Kluwer, Dordrecht (1993)

5. Milnor, J.: On the Betti numbers of real varieties. Proc. Amer. Math. Soc. 15, 275-280 (1964)

6. Logan, B., Shepp, L.: Optimal reconstruction of functions from its projections. Duke Math. 42, 645-659 (1975)

7. Maiorov, V.: On best approximation by ridge functions. J. Approx. Theory 99, 68-94 (1999)

8. Maiorov, V., Pinkus, A.: Lower bounds for approximation by MLP neural networks. Neurocomputing 25, 81-91 (1999)

9. Pinkus, A.: Approximation theory of the MLP model in neural networks. Acta Numer. 8, 143196 (1999)

10. Oskolkov, K.I.: Ridge approximation, Chebyshev-Fourier analysis and optimal quadrature formulas. Proc. Steklov Inst. Math. 219, 265-280 (1997)

11. Stein, E.M., Weiss, G.: Introduction to Fourier Analysis on Euclidean Spaces. Princeton University Press, Princeton (1971)

12. Vostrecov, B.A., Kreines, M.A.: Approximation of continuous functions by superpositions of plane waves. Dokl. Akad. Nauk SSSR 140,1237-1240 (1961) (Soviet Math. Dokl. 2, 1320-1329 (1961))

13. Vitushkin, A.G.: Estimation of the Complexity of the Tabulation Problem. Fizmatgiz, Moscow (1959)

14. Warren, H.E.: Lower bounds for approximation by nonlinear manifold. Trans. Amer. Math. Soc. 133, 167-178 (1968)

15. Zygmund, A.: Trigonometric Series, vol. 1. Cambridge University Press, Cambridge (1959) 